\title{
DETERMINACIÓN DE LA HUELla HÍDRICA PARA UN CULTIVO De guaYaba PERA (PSIDIUM GUajaVa)
}

\author{
Determination of the Water Footprint For \\ a Crop of Guava Pear (Psidium guajava)
}
Javier Hernando Corba Piraquive', Luis Javier Vargas Gómez², Jesús Alfonso Torres Ortega ${ }^{3}$

Centro Lasallista de Investigación en Modelación Ambiental - CLIMA, Universidad de la Salle, Bogotá, Colombia

Recibido: 23/04/2018 • Aprobado: 19/05/2018

\section{RESUMEN}

El guayabo, y su fruto, la guayaba, es una especie de pequeño árbol perteneciente a la familia de las mirtáceas. Este trabajo se desarrolló en cuatro fases de las cuales destacamos la primera y la tercera por corresponder a las etapas de toma y análisis de datos respectivamente. La fase inicial comprendió la revisión bibliográfica en la cual se tuvo en cuenta el reconocimiento del lugar, tipo de cultivo a estudiar y el sistema de riego adecuado para la elaboración del diagnóstico del cultivo así mismo del consumo de agua para el cultivo guayaba pera. Para los cálculos de la huella hídrica azul, verde y gris se aplicó la metodología del Manual de evaluación de la huella hídrica propuesto por Arjen Y. Hoekstra, donde fue necesario contar con la información de campo, datos como el área del cultivo, la cantidad de árboles sembrados, el método de riego y el rendimiento del cultivo. Para la tercera fase mediante los datos obtenidos por el modelamiento de los datos a través del software CropWat 8.0 se pudo determinar un valor de la huella hídrica total de cultivo con un valor de $0,55 \mathrm{~m} 3 /$ ton cuyo valor no alcanza a ser suministrado por la precipitación presentada en el lugar, debido a esto se realiza la captación de un cuerpo de agua cercano, actividad que afecta directamente a la población que vive aguas abajo del cultivo, ya que reduce en una forma considerable el caudal del caño en épocas de verano por lo que en la fase cinco se determinó que la mejor alternativa era la de implementar un pozo profundo para complementar la hidratación requerida de la planta.

Palabras clave: agua, diagnóstico, guayaba, hídrica, huella.

\section{Abstract}

The guava is a species of small tree belonging to the family of the Myrtaceae. This work was developed in five phases of which we emphasize the first and third to correspond to the stages of taking and analysis of data, respectively. The initial phase included the bibliographic review, which took into account the recognition of the place, type of crop for studding and the appropriate irrigation system for the preparation of the diagnosis of the crop as crop, as well as the consumption of water

\footnotetext{
${ }^{1}$ cjavier62@unisalle.edu.co, orcid.org/0000-0002-0260-4874

2 luisjvargas49@unisalle.edu.co, orcid.org/0000-0002-0297-7195

3 jatorres@unisalle.edu.co, orcid.org/0000-0002-4476-0816
} 
for the guava pear crop. For calculations of the blue, green and gray water footprint, the methodology of the Water Footprint Assessment Manual proposed by Arjen Y. Hoekstra was applied, where it was necessary to have field information, data such as the area of the crop, the amount of planted trees, irrigation method and crop yield. For the third phase, using the data obtained by modeling the CropWat 8.0 software, it was possible determine the total water footprint of culture with a value of $0.55 \mathrm{m3} / \mathrm{ton}$. As this value does is not supplied by the precipitation presented in the place, then is done by collecting a body of water nearby, this activity directly affects the population living downstream of the crop as it reduces in a considerable way the flow hydric in the summer. Then in phase five it was determined that the best alternative was to implement a deep well to supplement the hydration required of the plant.

Key words: diagnosis, guava, hydric, trace, water.

\section{INTRODUCCIÓN}

El árbol de guayabo (Psidium guajava) es un arbusto fuerte y resistente a enfermedades; su fruta es de color amarillo pálido cuando madura y tiene forma de pera, pesa en promedio 180 gramos, su pulpa puede ser blanca o rosada y es un árbol de gran producción y de amplios usos tanto medicinales como alimenticios (Alves et al., 2017; García, 2009; Jiao et al., 2017). Por otra parte, el concepto de huella hídrica nació como un indicador del uso del agua por Arjen Hoekstra, el concepto fue evolucionando y sus fórmulas de cálculo se fijaron en varias publicaciones elaboradas también por miembros de la United Nations Educational, Scientific and Cultural Organization - Unesco (Arévalo, Lozano \& Sabogal, 2011). El desarrollo de la evaluación en la huella hídrica en general, ayuda a la optimización de los procesos en los cuales se centran, también para comprender la disponibilidad de agua en el momento de realizar determinadas actividades, la necesidad de planificar un manejo de agua estructurado en la producción agrícola, siendo esta la actividad de mayor demanda de agua y por consiguiente lograr minimizar los impactos generados por un deficiente manejo de este recurso (Arévalo, 2012).

Para el proceso productivo de la guayaba pera se cuenta con una serie de impactos ambientales que afectan tanto al recurso agua, como al aire y suelo, pero por las consecuencias generadas la captación del recurso hídrico y la modificación del suelo son las que más implicadas se encuentran (Flores et al., 2015). Uno de los mayores impactos encontrados para el cultivo de guayaba es la captación de agua, ya sea superficial o subterránea, que es necesaria para las labores de riego del cultivo por cuanto se observa un uso excesivo del recurso, que se provoca por deficientes drenajes y la consecuente erosión, lo que conlleva a mediano plazo a la reducción de los cuerpos hídricos; otro de los impactos es el deterioro físico de los suelos, presentándose conflictos entre el uso del agua para los cultivos y el consumo humano (Casarotti et al., 2018).

Con el cálculo de la huella hídrica se determina la medición del volumen de agua total requerido para la producción del cultivo de guayaba pera, y con este indicador se da paso a buscar estrategias que propongan un mejor uso del recurso hídrico. El programa CropWAT 8.0 permite configurar proyectos de riego para diferentes condiciones de manejo y también se puede utilizar para evaluar las prácticas de riego de los agricultores, así como para estimar el rendimiento de los cultivos, tanto en condiciones de secano como de regadío.

\section{Metodología}

La investigación se desarrolló mediante cuatro fases como sigue: 


\subsection{Diagnóstico (Fase I)}

Esta fase se desarrolló mediante un enfoque cualitativo, que básicamente consistió en la recolección de los datos de las variables necesarias para realizar el modelamiento planteado para la segunda fase, también incluye el posicionamiento geográfico, en la cual se tendrá en cuenta el reconocimiento del lugar, el tipo de cultivo, tipo de riego y otros parámetros climatológicos para la elaboración del diagnóstico y determinar la situación actual de consumo de agua en el cultivo de guayaba pera.

\subsection{Modelamiento (Fase II)}

Esta fase consistió en realizar la cuantificación de la huella hídrica partiendo de los datos obtenidos en la fase I, para ello se tienen en cuenta las variables climáticas mencionadas en el marco teórico. Posterior a ello se introducen los valores de los datos requeridos por el software de modelamiento CropWAT 8.0 y así se obtendrá el cálculo de la huella hídrica del cultivo de guayaba pera.

\subsection{Análisis de resultados (Fase III)}

En esta fase se analizó el valor de la huella hídrica calculada en la Fase II para determinar si el consumo de agua asociado al cultivo de guayaba pera objeto de estudio, tiene tendencias a condiciones de sostenibilidad o por el contrario se encuentra en niveles de consumo inadecuado de este recurso.

\subsection{Formulación de alternativas (Fase IV)}

Dependiendo de los resultados obtenidos en el análisis se plantean alternativas que contribuyen al uso adecuado del recurso hídrico en la actividad agrícola que acompaña la producción frutícola de guayaba pera.

\section{Resultados}

\subsection{Diagnóstico (Fase I)}

En la Tabla 1 encontramos las principales generalidades del cultivo, con estas variables es posible realizar el cálculo de la huella hídrica azul que corresponde al uso del agua proveniente del riego, el cálculo de la huella hídrica verde, que representa el uso del agua proveniente de la precipitación y el cálculo de la huella hídrica gris, que incorpora el uso del agua requerido para la degradación de los agroquímicos usados en el cultivo.

TABLA 1

Generalidades del cultivo guayaba pera (Psidium guajava).

\begin{tabular}{|c|c|c|}
\hline \multirow{2}{*}{ Ubicación } & Latitud & Longitud \\
\hline & $3.34 \mathrm{~N}$ & $74.04 \mathrm{~W}$ \\
\hline \multirow{2}{*}{ Área } & \multirow{2}{*}{$\begin{array}{l}\text { La finca cuenta con un área total } \\
\text { de } 24 \text { hectáreas }\end{array}$} & 22 hectáreas de cultivo de guayaba pera (producción). \\
\hline & & $\begin{array}{l}2 \text { hectáreas de zonas duras que incluyen oficinas } \\
\text { administrativas, carreteras y el área de pos cosecha. }\end{array}$ \\
\hline \multirow[b]{2}{*}{ Producción } & \multicolumn{2}{|c|}{ Por cada hectárea encontramos 400 árboles sembrados de guayaba pera. } \\
\hline & \multicolumn{2}{|c|}{$\begin{array}{l}\text { En una hectárea la producción aproximada según información del administrador de la finca La Mariana es } \\
\text { de } 12.000 \text { canastillas anuales, el peso aproximado de cada canastilla es de } 22 \mathrm{~kg} \text {. Para una producción total } \\
\text { aproximada de } 264.000 \mathrm{~kg} / \text { año. Que corresponde a una producción de } 264 \text { toneladas de guayaba por año en la } \\
\text { totalidad del área sembrada. }\end{array}$} \\
\hline $\begin{array}{l}\text { Sistema de riego } \\
\text { del cultivo }\end{array}$ & \multicolumn{2}{|c|}{$\begin{array}{l}\text { El riego del cultivo se realiza por la aspersión donde la captación del agua se realiza de un cuerpo de agua } \\
\text { cercano y cuyo caudal no es muy grande. No se tiene clara la información del requerimiento hídrico del cultivo } \\
\text { de guayaba pera; por investigación se determinó que se debe proporcionar agua durante todos los periodos del } \\
\text { año, ya sea en invierno o en verano, hasta el momento no existen datos sobre requerimientos diarios de agua. }\end{array}$} \\
\hline
\end{tabular}

Fuente: autores. 
En esta fase se procedió a investigar acerca de la estación meteorológica más cercana al cultivo frutícola, y de la cual se utilizaron los datos recopilados para determinar mediante el programa CropWAT 8.0 las soluciones numéricas al problema de este proyecto. Los datos de la estación son los presentados en la Tabla 2.

TABLA 2

Datos de estación meteorológica del municipio de Lejanías (Meta)

\begin{tabular}{|c|c|}
\hline Nombre de la estación & Lejanías - Castillo \\
\hline País & Colombia \\
\hline Código & 32060090 \\
\hline Latitud & $3.34 \mathrm{~N}$ \\
\hline Longitud & $74.03 \mathrm{~W}$ \\
\hline Altitud & 840 \\
\hline
\end{tabular}

El cultivo se encuentra localizado en Lejanías (Meta), este municipio cuenta con un clima tropical, el cual lo hace lluvioso en la mayoría de meses del año, según la clasificación climática de Köppen-Geiger a este municipio se le da la categorización que indica que ningún mes tiene precipitación por debajo de $60 \mathrm{~mm}$ y se considera cálido y lluvioso todo el año, la precipitación que se presenta durante el ańo es elevada respecto a otros lugares del país.

\subsection{Modelamiento (Fase II)}

El programa CropWAT 8.0 es un programa informático para el cálculo de los requerimientos de agua de los cultivos y las necesidades de riego con base en datos de suelo, clima y la evapotranspiración (Cleves et al., 2016). Con los datos obtenidos en la Fase I se procede a realizar el modelamiento utilizando el software CropWAT 8.0 con el fin de poder determinar la huella hídrica verde, la huella hídrica azul, la huella hídrica gris y la huella hídrica total. Se procedió siguiendo técnicas similares a las usadas en trabajos anteriores (Carrillo y Chavarro, 2015; Fonseca, Lizarazo \& Torres, 2016; Sánchez et al., 2015).

A continuación, se explica brevemente cómo funciona el programa y los datos requeridos por el mismo. En la tabla 3 se muestran los datos incluidos en el módulo clima/evapotranspiración (ETo) respecto a la ubicación geográfica donde se desarrolla el cultivo. Posterior a ello, con la información obtenida de la estación climatológica para los parámetros de temperatura mínima, temperatura máxima, humedad, viento e insolación se realiza un promedio para cada uno de ellos durante los 5 últimos años y mediante una hoja de Excel se vinculan al software. Finalmente, al correr esta primera parte del modelo y si los datos suministrados son incluidos de forma correcta, este módulo nos arrojará los datos de radiación solar estimada, que llega a la superficie del suelo y la evapotranspiración de referencia que se evidencian en las columnas resaltadas con color amarillo.

El objetivo de este módulo es poder encontrar el valor promedio de evapotranspiración, que para este caso es de $5,56 \mathrm{~mm} \mathrm{H}_{2} \mathrm{O} /$ día, y cuyo dato es indispensable para realizar el cálculo de la huella hídrica. La ETo se puede determinar mensual, decenal y diariamente, dependiendo del requerimiento de los autores. El segundo módulo del software comprende la precipitación cuyos datos también fueron obtenidos por medio de la estación climatológica ubicada cerca al lugar del cultivo, para ello también se realizó el promedio de los últimos 5 años, y, posteriormente, estos datos se introdujeron en este módulo, también se eligió el tipo de método para el cálculo de la precipitación efectiva, para este caso utilizamos un método por defecto incluido en el software. 
Tabla 3

Evapotranspiración

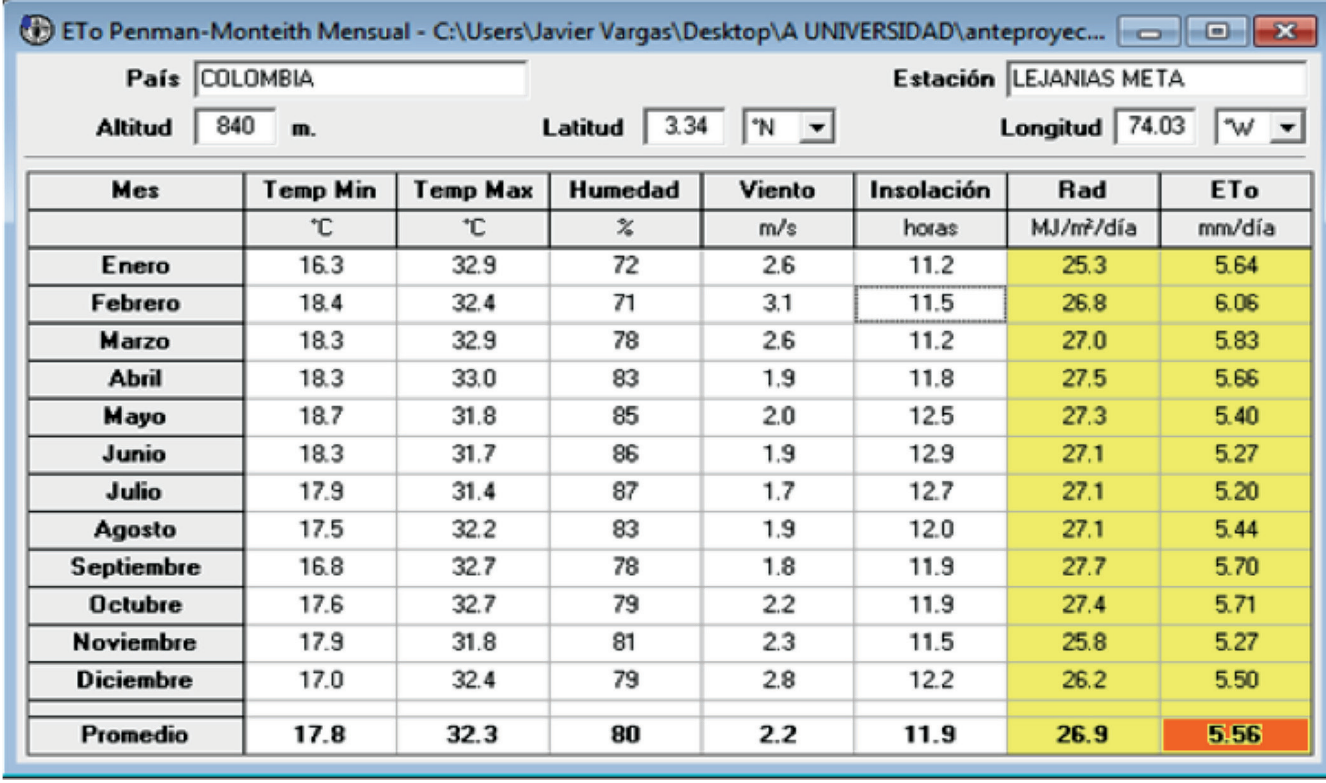

Fuente: software CropWAT 8.0

TABla 4

Precipitación

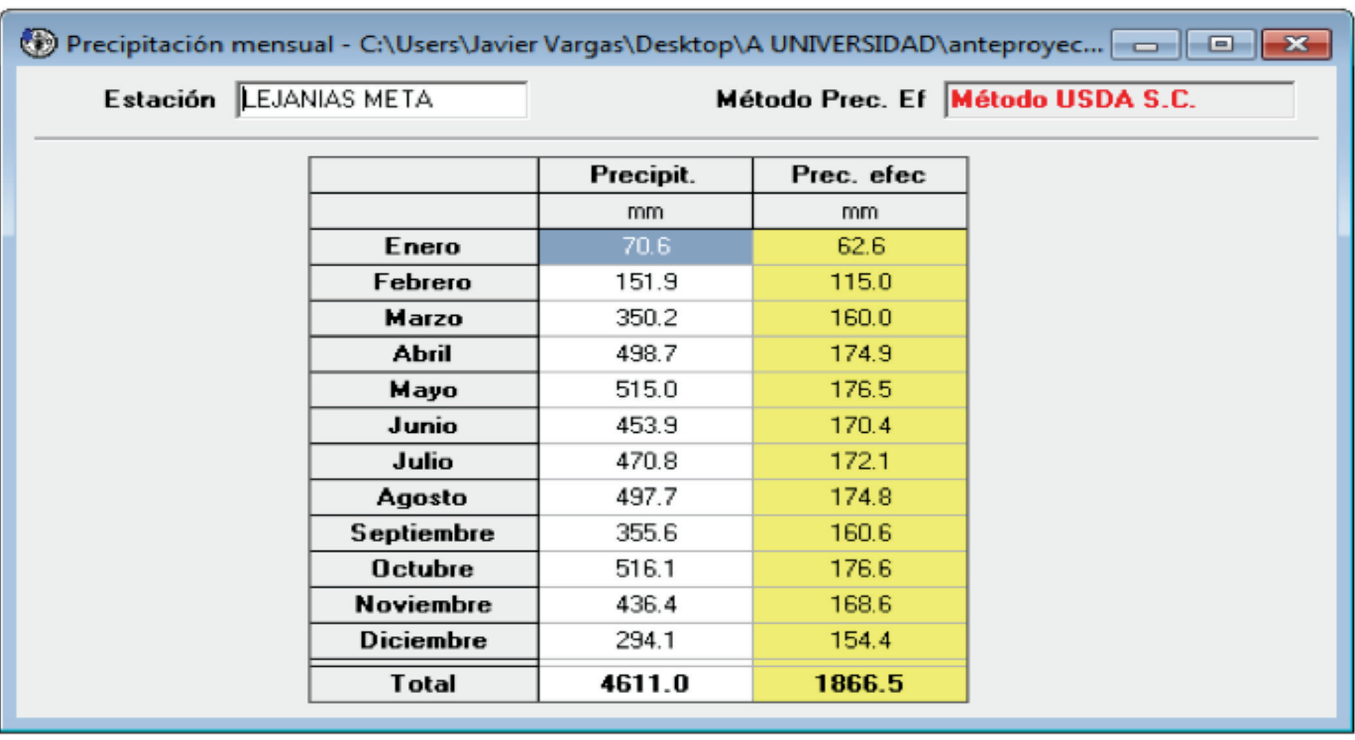

Fuente: software CropWAT 8.0. 


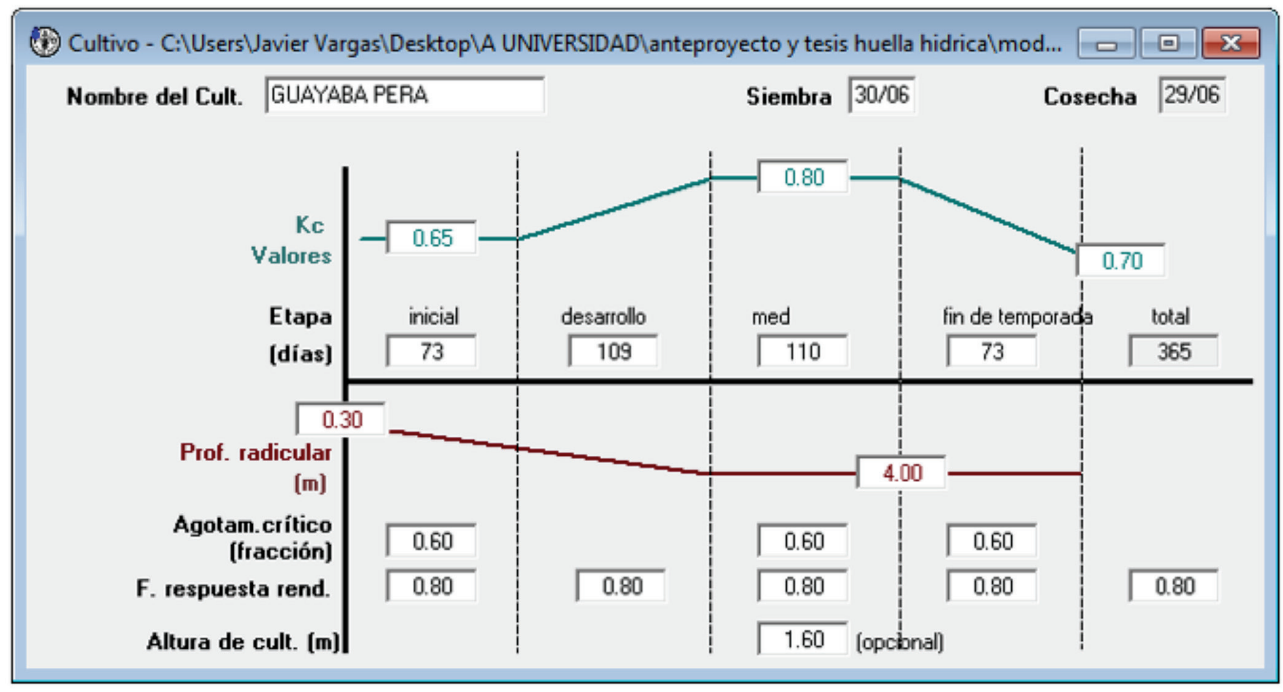

Fig. 1. Características del cultivo de guayaba pera.

Fuente: software CropWAT 8.0.

En la tabla 2, se muestran los datos incluidos en el módulo de precipitación, posterior a ellos se corre el segundo módulo, para finalmente obtener los datos de precipitación efectiva, que se refiere a la parte de la precipitación que puede ser efectivamente utilizada por las plantas, ya que no toda la precipitación está disponible para el requerimiento del cultivo. El objetivo de este módulo es encontrar el valor total de precipitación efectiva, que para este caso es de 1866.5 $\mathrm{mm} \mathrm{H}_{2} \mathrm{O}$ cuyo valor también es indispensable para el cálculo de la huella hídrica verde y que será utilizado más adelante.

El siguiente paso fue determinar qué tiempo es el que relaciona completamente la profundidad radicular y el coeficiente del cultivo. Los datos necesarios en este paso fueron investigados por el equipo de trabajo ya que en la base de datos de este programa no se encontró información sobre el cultivo que estamos trabajando.

En la Figura 1 se muestra el tercer módulo del software que comprende las características del tipo de cultivo, los datos requeridos para este módulo fueron el nombre del cultivo, las fechas de siembra y cosecha, los valores del coeficiente del cultivo Kc que comprende el consumo de agua o la evapotranspiración que ocurre en una superficie cultivada.

Posteriormente, se incluyen los valores en cuanto a los días en los cuales se desarrollan las etapas de dicho cultivo a lo largo del año; dicha información fue suministrada por el encargado del cultivo. Seguido de ello se introducen los valores de la profundidad radicular de la planta que indica la capacidad del cultivo para aprovechar la reserva de agua del suelo. Finalmente, el modelo arroja los valores de agotamiento crítico y el factor de respuesta del rendimiento y la altura del cultivo que es un valor opcional dentro del modelo.

Para el cuarto módulo del software, se requiere el nombre del suelo y las características del mismo, es indispensable contar con esta información para que se pueda desarrollar con normalidad el modelamiento. Para nuestro caso, fue investigado el tipo de suelo que se presente donde se desarrolla el cultivo, mediante el Plan de Ordenamiento Territorial (POT) se pudo determinar que el suelo presentado en el lugar es de tipo arcilloso de coloración negra. Para este módulo, en la base de datos que nos ofrece el software, se pudo encontrar este mismo tipo de suelo Black Clay Soil, y, que por defecto nos arroja los datos generales del suelo 
requeridos por el programa. Finalmente, el quinto módulo del software, al cual se llega después de completar la información requerida en los módulos anteriores, se puedo determinar el requerimiento de agua del cultivo, el cual comprende la cantidad de agua necesaria para compensar la pérdida de evapotranspiración del campo de cultivo.

En la Tabla 5, se pueden ver los valores arrojados por el modelamiento del software en los cuales se indican los valores del coeficiente del cultivo, la evapotranspiración decanal y por días, la precipitación efectiva y finalmente el requerimiento de riego del cultivo cuyo valor es de $119,5 \mathrm{~mm} \mathrm{H}_{2} \mathrm{O} / \mathrm{dec}$ y que es indispensable para realizar el cálculo de la huella hídrica azul del cultivo. Una vez que se tuvo definido cada uno de los datos necesarios para poder resolver las ecuaciones mencionadas por Hoekstra et al. (2011), se procedió a resolverlas para determinar la huella hídrica en el proceso de cultivo guayaba pera.

TABla 5.

Características del sustrato

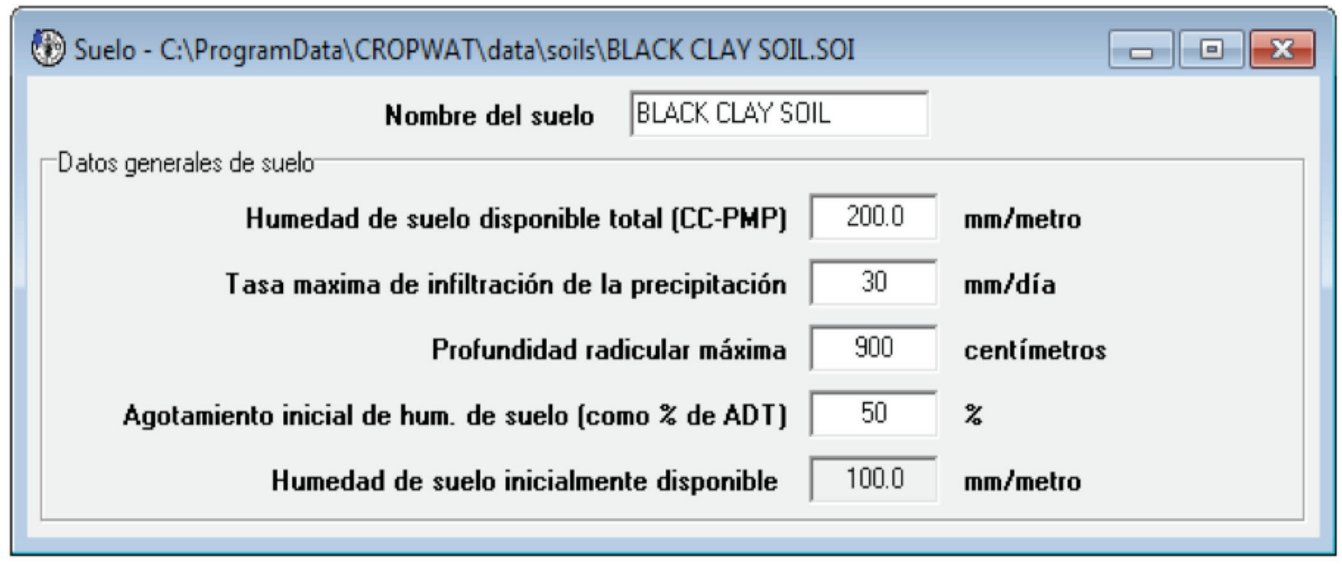

Fuente: Software Cropwat 8.0.

\section{Huella hidrica azul HHA}

Para el cálculo de la huella hídrica azul utiliza la siguiente ecuación:

$$
H H A=\left(\frac{c w u}{Y}\right){ }^{*} \# \text { de hectareas }=\text { vol/masa }
$$

En la cual encontramos los siguientes datos requeridos:

CWU: que corresponde al requerimiento de agua del cultivo y cuyo valor fue determinado mediante el modelamiento del software en el módulo 5, el cual trata de la determinación del requerimiento de agua del cultivo, dicho valor corresponde a 119,5 mm/década.

$Y$ : corresponde al rendimiento del cultivo expresado en toneladas/ha. Para este caso encontramos que la producción de guayaba pera corresponde a 12.000 canastillas por año/ha, el peso aproximado de cada canastilla es de $22 \mathrm{~kg}$. Como la formula requiere el rendimiento en toneladas por hectárea realizamos la siguiente operación.

$$
\begin{gathered}
Y=12.000 \text { canastillas } / \mathrm{ha}{ }^{*} 22 \mathrm{~kg} / \text { canastilla } \\
=264.000 \mathrm{~kg} / \mathrm{ha}
\end{gathered}
$$

Área de captación $=22$ hectáreas área de producción de guayaba pera 


$$
\begin{array}{r}
H H A=\left(\frac{119,5 \frac{\mathrm{mm}}{\mathrm{dec}} * \frac{1 \mathrm{dec}}{10 a \tilde{\mathrm{n} o s}} \mathrm{~m}^{2}}{264 \frac{\text { ton }}{\mathrm{ha}}}\right) \\
=\frac{\left(11,95 \mathrm{~mm} * \frac{1 \mathrm{~m}}{1000 \mathrm{~mm}} * 1 \mathrm{~m}^{2}\right)}{264 \frac{\text { ton }}{\mathrm{ha}}} \\
/ 22 \mathrm{ha}
\end{array}
$$

$$
H H A=0,000000205 \frac{m^{3}}{\text { ton }}
$$

\section{Huella hidrica verde $H H V$}

Para el cálculo de la huella hídrica verde se tiene en cuenta la siguiente ecuación.

$$
H H V=\left(\frac{\text { PRECe }}{Y}\right) * \# \text { de hectareas }=\mathrm{vol} / \text { masa }(2)
$$

En la cual encontramos los siguientes datos requeridos:

PRECe: corresponde a la precipitación en $\mathrm{mm}$ y cuyo valor fue determinado mediante el software en módulo de precipitación, dicho valor corresponde a $1866,5 \mathrm{~mm}$.

$Y$ : rendimiento del cultivo expresado en ton/ha para este caso ya se había determinado este valor teniendo como resultado, 264 ton/ha.

Área de captación = esta área corresponde al área de producción frutícola que corresponde a 22 hectáreas.

$$
\begin{array}{r}
H H V=\left(\frac{1866,5 \mathrm{~mm}}{264 \frac{\text { ton }}{\mathrm{ha}}}\right) \\
=\frac{\left(1866,5 \mathrm{~mm} * \frac{1 \mathrm{~m}}{1000 \mathrm{~mm}} * 1 \mathrm{~m}^{2}\right)}{264 \frac{\text { ton }}{\mathrm{ha}}} \\
/ 22 \mathrm{ha} \\
H H V=0,000321 \frac{\mathrm{m}^{3}}{\text { ton }}
\end{array}
$$

$$
H H G=\left(\frac{\frac{(\alpha * \mathrm{AR})}{(\mathrm{Cmax}-\mathrm{Cnat})}}{Y}\right)=\frac{\text { vol }}{\text { masa }}(3)
$$

Huella hidrica gris $H H G$

Para el cálculo de la huella hídrica gris se tiene en cuenta la siguiente ecuación.

Para el cálculo de la huella hídrica gris se deben tener en cuenta los siguientes valores.

$\alpha$ : La fracción de lixiviación corresponde a las sustancias químicas aplicadas a los cultivos que, en el caso de no tener los datos de medición, se debe tomar según el manual de Hoekstra con un valor de $10 \%$. (Ercin, Mekonnen, \& Hoekstra, 2013).

AR: cantidad aplicada de producto químico para el campo por hectárea (kg/ha).

Cmax: concentración máxima aceptable de productos químicos aplicados en el cultivo.

Cnat: es la concentración natural de los contaminantes.

$Y$ : rendimiento del cultivo expresado en toneladas/año.

Área de captación: es el área total de la producción frutícola de la finca, para este caso son 22 hectáreas.

Para el cálculo de la huella hídrica gris se tuvieron en cuenta los fertilizantes utilizados en $\mathrm{kg} / \mathrm{Ha}$ (Baraona \& Sancho, 2001). Cabe mencionar que los productos químicos utilizados en el cultivo como pesticidas, herbicidas, insecticidas y demás, no se tuvieron en cuenta debido a la falta de precisión y exactitud en las cantidades promedio que se agregan al cultivo de guayaba pera. En la Tabla 6 se muestran los fertilizantes utilizados en el cultivo y el peso promedio aplicado por hectárea.

Para determinar la concentración máxima permitida, se investigó por parte de los autores, pero la información encontrada en cuanto a legislación de vertimientos para uso agrícola es muy básica, teniendo en cuenta el lugar de estudio donde se desarrolla el cultivo. Para este caso en particular se utilizó el acuerdo 43 del 17 de octubre de 2006 de la Corporación 
Autónoma Regional de Cundinamarca, en que se proponen los objetivos de calidad del agua para la cuenca del río Bogotá a lograr en el año 2020, allí se establecen los valores máximos aceptables de los productos químicos que se utilizan en diversos cultivos; este acuerdo lo utilizamos como referencia, ya que a pesar que corresponde a una corporación regional ajena al lugar donde se desarrolla el proyecto, esta hace parte de la legislación colombiana.

\section{TABLA 6}

Fertilizantes para el cultivo de guayaba pera

\begin{tabular}{|c|c|c|}
\hline Fertilizante & $\begin{array}{c}\text { Promedio tasa } \\
\text { aplicación de } \\
\text { fertilizante, } \mathbf{k g} / \mathbf{h a}\end{array}$ & $\begin{array}{c}\text { Concentración en } \\
\text { el agua (máximo } \\
\text { aceptable), mg/L }\end{array}$ \\
\hline $\begin{array}{c}\text { Sulfato } \\
\text { de amonio }\end{array}$ & 293 & 400 \\
\hline $\begin{array}{c}\text { Nitrato } \\
\text { de calcio }\end{array}$ & 308 & 10 \\
\hline $\begin{array}{c}\text { Sulfato } \\
\text { de potasio }\end{array}$ & 120 & 400 \\
\hline
\end{tabular}

Fuente: ICA (2012)

Al tener el promedio de la tasa de aplicación del fertilizante y conocer la concentración máxima permitida, se requiere el factor de lixiviación; debido a la falta de datos específicos al cultivo sobre el porcentaje de lixiviación hasta alcanzar agua dulce se asumirá el valor utilizado por Hoekstra de $10 \%$ para todos los fertilizantes para la concentración natural del contaminante que se refiere a la concentración en el cuerpo de agua que podría ocurrir sin intervención humana, a falta de información se asume que este valor es cero ya que es la concentración del producto químico presente en una fuente de agua natural (Doorenbos et al., 1979; FAO, 2013).

Huella hidrica gris para sulfato de amonio

$$
\begin{aligned}
& \alpha=0,10 ; \mathrm{AR}=293 \mathrm{~kg} / \mathrm{ha} ; \mathrm{C} \max =400 \mathrm{mg} / \mathrm{L} \\
& \text { Cnat }=0 ; \mathrm{Y}=264 \mathrm{ton} / \mathrm{ha} ;
\end{aligned}
$$

$\operatorname{ton} / \mathrm{m}^{3}$
Huella hidrica gris para nitrato de calcio

$\alpha: 0,10 ; A R=308 \mathrm{~kg} / \mathrm{ha}$; Cmax: $10 \mathrm{mg} / \mathrm{L}$;

Cnat $=0 ; \mathrm{Y}=26,4$ ton $/$ ha

$$
\begin{aligned}
& \mathrm{cmax}=10 \frac{\mathrm{mg}}{\mathrm{L}} * \frac{1000 \mathrm{~L}}{1 \mathrm{~m}^{3}} * \frac{1 \text { ton }}{10^{9} \mathrm{mg}}=10^{-5} \frac{\text { ton }}{\mathrm{m}^{3}} \\
& H H G=\left(\frac{\frac{(0,10 * 120 \mathrm{~kg} / \mathrm{ha})}{0,0004 \mathrm{mg} / \mathrm{L}-0}}{264 \text { ton } / \mathrm{ha}}\right)=11,66 \mathrm{~m}^{3} / \text { ton }
\end{aligned}
$$

Huella hidrica gris para el sulfato de potasio

$\alpha: 0,10 ; \mathrm{AR}=120 \mathrm{~kg} / \mathrm{ha} ; \mathrm{Cmax}=400 \mathrm{mg} / \mathrm{L}$; Cnat

$=0 ; \mathrm{Y}=26,4$ ton $/ \mathrm{ha}$

$$
\mathrm{Cmax}=400 \frac{\mathrm{mg}}{\mathrm{L}} * \frac{1000 \mathrm{~L}}{1 \mathrm{~m}^{3}} * \frac{1 \text { ton }}{10^{9} \mathrm{mg}}
$$

Huella hidrica gris total

HHGT = HHG Sulfato de amonio + HHG

nitrato de calcio+ HHG Sulfato de potasio

HHGT $=0,277 \mathrm{~m}^{3} /$ ton $+11,66 \mathrm{~m}^{3} /$ ton

$+0,114 m^{3} /$ ton

$\mathrm{HHGT}=12,051 \mathrm{~m}^{3} /$ ton

El cálculo final de la huella hídrica gris se divide por el total del área de producción de guayaba, ya que se está trabajando en relación a una hectárea en el cálculo de las huellas anteriormente calculadas.

HHGT/ $22 \mathrm{Ha}=\left(\frac{12,051 \mathrm{~m}^{3}}{\text { ton }}\right)\left(\frac{1 h a}{22 h a}\right)=0,548 \mathrm{~m}^{3} / \mathrm{ton}$

\section{4 Huella hídrica total}

Finalmente, una vez que se obtuvieron los datos concretos sobre las tres huellas hídricas individualizadas, se procedió a resolver la ecuación final que se ve a continuación.

$$
\begin{gathered}
H H \text { total }=\mathrm{HHV}+\mathrm{HHA}+\mathrm{HHG}=\text { vol } / \text { masa }(4) \\
\begin{aligned}
H H T=0,000321 & +0,000000205+0,548 \\
= & 0,55 \frac{\mathrm{m}^{3}}{\text { ton }}
\end{aligned}
\end{gathered}
$$

\subsection{Análisis de resultados (Fase III)}

Mediante la recopilación de los datos meteorológicos requeridos por el programa y su posterior 
modelamiento como se evidencia en las fases uno y dos se obtuvo el cálculo de la huella hídrica total para el cultivo de guayaba pera obteniendo un valor de 0,55 $\mathrm{m}^{3} /$ ton la cual da cuenta del requerimiento de agua para producir una tonelada de guayaba pera en condiciones meteorológicas dadas por la ubicación geoespacial del lugar. Para llegar a este resultado fue necesario realizar el modelamiento del software CropWAT 8.0 y completar cada uno de los módulos para obtener este resultado fino.

La Huella hídrica verde, nos indica la cantidad de agua tomada por el cultivo del suelo superficial, en la cual, al despejar la fórmula con las siguientes variables; la precipitación presentada en el lugar, el rendimiento del cultivo y el área, obtuvimos el siguiente valor: $0,000321 \mathrm{~m}^{3} /$ ton. Con este resultado evidenciamos que la precipitación obtenida en el lugar no alcanza a suplir las necesidades de riego de todo el cultivo, por ello se hace la compensación necesaria realizando la captación de un acuífero. La eficiencia de la precipitación presentada en el lugar, según el modelo es de $69,7 \%$, presentando una precipitación total de 4611,0 $\mathrm{mm}$, precipitación efectiva de 3209,6 mm y una pérdida total de $14,01 \mathrm{~mm}$.

El complemento del requerimiento hídrico del cultivo se realiza por la captación de un acuífero cercano, para este caso realizamos el cálculo de la huella hídrica azul, que indica la cantidad de agua requerida por el cultivo tomada de agua superficial o subterránea. Al despejar la formula con las siguientes variables: uso de agua del cultivo, rendimiento del cultivo y el área obtuvimos el siguiente valor: $2,05 \times 10^{-6} \mathrm{~m}^{3} /$ ton. Encontramos que el requerimiento, aunque es menor respecto a la huella hídrica verde, es indispensable para que la producción del cultivo sea la óptima y los resultados sean los esperados.

La huella hídrica gris nos indica el agua que se requiere para asimilar la carga de contaminantes del cultivo. para este caso se tuvo en cuenta tres tipos de fertilizantes, para cada uno se tuvieron en cuenta las siguientes variables, la fracción de lixiviación, cantidad aplicada de producto químico para el campo por hectárea, concentración máxima aceptable de productos aplicados, concentración natural del contaminante, rendimiento del cultivo, se realizó el cálculo para cada uno de ellos y posteriormente se realizó la sumatoria obteniendo un valor de $0,458 \mathrm{~m}^{3} /$ ton.

\subsection{Formulación de alternativas (Fase IV)}

La herramienta fundamental fue el software, ya que mediante este se pudo determinar la huella hídrica azul, la huella hídrica verde, la huella hídrica gris para finalmente determinar la huella hídrica total. Dicha herramienta permite complementar información hidrogeológica muy importante que fue investigada por los autores, para conseguir que el modelo funcione correctamente, y con esto finalmente realizar el despeje de las fórmulas y determinar los valores de las huellas los cuales se evidencian a continuación.

Para la formulación de esta alternativa, fue indispensable cada una de las fases anteriores, ya que eran el paso a paso para plantear una propuesta precisa, la cual contribuya a la mitigación de impactos ambientales, la reducción de la captación de agua de los cuerpos hídricos, y el aprovechamiento de los recursos hidrogeológicos.

A pesar de que la precipitación presentada durante el año es elevada, respecto a otros lugares del país, no alcanza a suplir la necesidad del cultivo, en cuanto a requerimiento de recurso hídrico se refiere, ya que según la información encontrada, para este cultivo en particular, es indispensable que en todas las épocas de año, ya sea invierno o verano, la siembra debe tener agua continuamente, por lo que la variabilidad en la precipitación puede influenciar en la captación y el riego ya que se realiza de un medio natural, el cual está sujeto a la variación del caudal, en cuanto a épocas del año y la reducción del mismo por actividades humanas que se puedan realizar río arriba de donde se realiza la captación.

En la Tabla 7 se determinó que las mayores reservas del país se encuentran en las provincias de los Llanos orientales, sin embargo, Cauca-Patía y la Cordillera Oriental son las provincias con mayor 
aprovechamiento, suficientes para suplir las necesidades actuales.

\section{TABLA 7}

Provincias hidrogeológicas

\begin{tabular}{|c|c|}
\hline $\begin{array}{c}\text { Provincia } \\
\text { hidrogeológica }\end{array}$ & $\begin{array}{c}\text { Llanos } \\
\text { Orientales }\end{array}$ \\
\hline $\begin{array}{c}\text { Formaciones geológicas con } \\
\text { potencial hidrogeológico }\end{array}$ & $\begin{array}{c}\text { Mirador Guayabo/Caja, } \\
\text { Necesidad, Corneta, depósitos } \\
\text { recientes de tipo aluvial } \\
\text { y terrazas. }\end{array}$ \\
\hline Tipo de acuífero & $\begin{array}{c}\text { Acuíferos libres a } \\
\text { semiconfinados en los } \\
\text { sedimentos aluviales }\end{array}$ \\
\hline Área (km $\left.{ }^{2}\right)$ & $265.547,29$ \\
\hline Espesor acuífero (m) & 550 \\
\hline Rendimiento específico & $0,02-0,08$ \\
\hline Reservas (m ${ }^{3 * 1000)}$ & 239,06 \\
\hline
\end{tabular}

Fuente: Corba y Vargas (2018).

La importancia de la sostenibilidad y la preservación de nuestros cuerpos de agua, nos llevan a plantear la alternativa de poder darle aprovechamiento al recurso hídrico encontrado en las reservas hidrogeológicas que hacen parte de nuestro territorio. El 74,5\% del territorio nacional está cubierto por provincias hidrogeológicas y solo el $25,5 \%$ por rocas ígneas, metamórficas o por ambientes con posibilidades hidrogeológicas desconocidas, limitadas o restringidas, lo cual indica por sí solo una riqueza de recurso hídrico. En el caso de Lejanías, es uno de los municipios que hacen parte del departamento del Meta, el cual está ligado a la provincia hidrogeológica de los Llanos Orientales.

En la Tabla 8 se puede encontrar el aprovechamiento que le da cada zona hidrogeológica del país, al recurso hídrico subterráneo, en donde se evidencia que la provincia hidrogeológica con mayor consumo es la de Cauca-Patía.

La mayoría de acuíferos constituidos en esta región, tienen conexión hidráulica con los ríos principales en algunos puntos, y en otros reciben aporte de precipitación, en los sitios donde afloran las unidades de aluviones. La provincia hidrogeológica de los Llanos Orientales cuenta con el mayor volumen de agua subterránea sobre la diferente provincia hidrogeológica del país.

TABLA 8

Distribución del uso del agua subterránea por provincia hidrogeológica

\begin{tabular}{|c|c|c|}
\hline Provincia & $\begin{array}{c}\text { Uso millones } \mathbf{~ m}^{3} / \\
\text { año }\end{array}$ & $\begin{array}{c}\text { Porcentaje } \\
\text { nacional }\end{array}$ \\
\hline Cauca-Patía & 420 & $50 \%$ \\
\hline Cordillera Oriental & 266 & $32 \%$ \\
\hline Sinú-San Jacinto & 27 & $3 \%$ \\
\hline Guajira & 22 & $3 \%$ \\
\hline Urabá & 21 & $3 \%$ \\
\hline $\begin{array}{c}\text { Valle bajo } \\
\text { del Magdalena }\end{array}$ & 14 & $2 \%$ \\
\hline Llanos Orientales & 24 & $3 \%$ \\
\hline Catatumbo & 30 & $4 \%$ \\
\hline
\end{tabular}

Fuente: Corba y Vargas (2018).

Ya que nuestro predio se encuentra en la región hidrogeológica, anteriormente explicada, y cuya composición de suelo, según el EOT, es de sedimentos mixtos aluviales con contenidos de cantos y gravas poco alteradas, cuyas características dan cuenta de una porosidad generosa, la cual facilita el trasporte del agua, la presencia de un nivel freático, considerado como alto, da para que en el predio estudiado se realicen perforaciones para llegar al nivel freático donde se encontrarán los acuíferos confinados, y se realice la construcción de aljibes para, finalmente, dar aprovechamiento al recurso hídrico del subsuelo y suplir la necesidad del cultivo.

También se acompaña esta alternativa con el cambio de sistema de riego, que actualmente se realiza por aspersión, el cual lleva un mayor consumo de agua que el método de riego gota a gota, el cual 
aporta ventajas como optimización del recurso hídrico, siendo un sistema que se implementa a largo plazo, se puede tener un control de abastecimiento por planta y finalmente facilita el control de plagas (Ayers \& Westcot, 1985).

Finalmente, un sistema de riego es sencillo de realizar, considerando la captación del recurso hídrico subsuperficial, y, por medio de una motobomba se puede realizar la extracción del agua que posteriormente se confinará en los aljibes para finalizar con su distribución. Con estudios más avanzados se determinará la cantidad de perforaciones, la ubicación y el montaje de los aljibes, así como la cantidad de agua a distribuir y demás detalles.

\section{Conclusiones}

Con el estudio se pudo formular una estrategia de reducción de la captación de agua que se hace de la cuenca aledańa al predio, para así aprovechar los recursos hidrogeológicos que muchas veces son despreciados.

Con la utilización del software CropWAT 8.0 se pudieron determinar los valores de huella hídrica verde 0,0032 $\mathrm{m}^{3} /$ ton, la huella hídrica azul: 0,000000205 $\mathrm{m}^{3} /$ ton y finalmente el cálculo de la huella hídrica gris: $0,458 \mathrm{~m}^{3} /$ ton para la producción de una tonelada de la fruta.

Al realizar el cálculo de la huella hídrica verde, azul y gris y su posterior suma se determinó la huella hídrica total, obteniendo un valor de $0,55 \mathrm{~m}^{3}$ por tonelada de guayaba pera.

La alternativa propuesta para implementar un pozo profundo, para complementar la hidratación requerida de la planta, contribuye a la reducción del impacto ambiental generado por la captación que se realiza de un cuerpo de agua superficial.

\section{REFERENCIAS}

Alcaldía de Lejanías (2016). Municipio de Lejanías. Información general. Recuperado en: http://www.lejanias-meta.gov.co/informacion_general.shtml\#geografia

Alves, L., Ferreira, G., Rodrigues, W., da Silva, T.,

Menini, L., Ferreira, A. \& da Silva, M. (2017). Larvicidal effect of essential oils from Brazilian cultivars of guava on Aedes aegypti L. Industrial Crops and Products, 108 (1), 684-689. https://doi. org/10.1016/j.indcrop.2017.07.034

Arévalo, D. (2012). Una mirada a la agricultura de Colombia desde su huella hídrica. WWF Reporte Colombia. Recuperado en: http://www.huellahidrica.org/Reports/Arevalo-2012-HuellaHidricaColombia.pdf

Arévalo, D., Lozano, J. \& Sabogal, J. (2011). Estudio nacional de huella hídrica Colombia- Sector Agrícola. Revista internacional de sostenibilidad, tecnología y humanismo, (7), 101-126. Recuperado en: http://www.huellahidrica.org/Reports/Articulo\%20 Huella\%20Hidrica\%20Colombia\%20publicado.pdf

Ayers, A. \& Westcot, D. (1985). Water quality for agricultura. FAO Irrigation and Drainage Paper. 29 Rev. 1. Food and Agriculture Organization of the United Nations. Rome, Italy. Recuperado en: http://www.fao.org/docrep/003/T0234E/T0234E00.htm

Baraona, M. \& Sancho, E. (2001). Fruticultura general. San José: Editorial Universidad Estatal a Distancia. Recuperado en: https://books.google.com.co/books?id=F5XZYgrK4WIC\&pri $\mathrm{ntsec}=$ frontcover $\& \mathrm{dq}=$ fruticultura + general $\& \mathrm{hl}=\mathrm{es}-419 \& \mathrm{sa}=\mathrm{X}$ \&ved=0ahUKEwj6x5SI3svNAhUDMSYKHS1PDyMQ6AEI

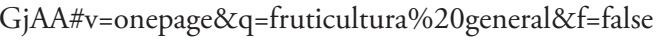

Bejarano González, A. \& Bello, R. (2003). Pymes de producción y comercialización de guayaba pera en Colombia. Recuperado en: http://intellectum.unisabana.edu.co/bitstream/handle/10818/6466/126564.pdf?sequence $=1$

Carrillo, A. \& Chavarro, D. (2015). Evaluación de la huella hídrica para dos ańos productivos de lima ácida Tahití en tres huertos de Corpoica. Trabajo de grado, Facultad de Ingeniería. Bogotá: Universidad de La Salle. Recuperado en: http://repository.lasalle.edu.co/handle/10185/18270

Casarotti, S., Borgonovi, T., Batista, C. \& Penna, A.

(2018). Guava, orange and passion fruit by-products: Characterization and its impacts on kinetics of acidification and properties of probiotic fermented products. LWT, (98), 69-76. https://doi. org/10.1016/j.lwt.2018.08.010

Cleves, J., Toro, J. \& Martínez, L. (2016). Los balances hídricos agrícolas en modelos de simulación agroclimáticos. Una revisión analítica. Revista Colombiana de Ciencias Hortícolas, 10(1), 149-163. 
Corba, J. \& Vargas, L. (2018). Evaluación de la huella hídrica para la producción de guayaba pera en el municipio de Lejanías, departamento del Meta. Trabajo de grado. Bogotá: Universidad de La Salle.

Chapagain, A., \& Hoekstra, A. (2004). Water footprints of nations. Volume 1: Main Report. Enschede: Unesco- IHE.

Doorenbos, J., Kassam, A., Bentvelsen, C., Branscheid, J., Plusje, J., Smith, M., Uittenbogaard, G. \& van der Wal, H. (1979). Efectos del agua sobre el rendimiento de los cultivos. Estudio FAO Riego y Drenaje 33. Roma: FAO.

Ercin, A. E., Mekonnen, M. M. \& Hoekstra, A. Y. (2013). Sustainability of national consumption from a water resources perspective: The case study for France. Ecological Economics, (88), 133-147. https://doi.org/10.1016/j.ecolecon.2013.01.015

FAO. (2013). Land and Water. Recuperado en: http://www.fao. org/nr/water/infores_databases_cropwat.html

Flores, G., Shi, W., Negrim, A. \& Kennelly, E. (2015).

Chemical composition and antioxidant activity of seven cultivars of guava (Psidium guajava) fruits. Food Chemistry. Volume $170(1), 327-335$.

Fonseca, 0., Lizarazo, J. \& Torres, J. (2016). Determinación de la huella hídrica del cultivo de algodón. Publicaciones e Investigación, (10), 39-46.
García, M. (2009). Guía técnica del cultivo de la guayaba. Ciudad Arce: Centro Nacional de Tecnología Agropecuaria y Forestal "Enrique Álvarez Córdova”.

Hoekstra, A. Y., Chapagain, A. K., Aldaya, M. M., \& Mekonnen, M. (2011). The Water Footprint Assessment Manual, London: Earthscan.

Instituto Colombiano Agropecuario - ICA (2012). Manejo fitosanitario del cultivo de guayaba (Psidium guajava, L.). Bogotá: ICA.

Jiao Y., Zhang M., Wang S. \& Yan C. (2017).

Consumption of guava may have beneficial effects in type 2 diabetes: $A$ bioactive perspective. International Journal of Biological Macromolecules. (101), 543-552.

Ministerio de la Agricultura (2011). Instructivo técnico para el cultivo de la guayaba. Instituto de Investigaciones en Fruticultura Tropical Asociación Cubana de Técnicos Agrícolas y Forestales. Recuperado en: http://www.innovacion.gob.sv/inventa/attachments/article/4330/index.pdf

Sánchez, E., Villareal, J. \& Torres, J. (2015). Estimación de la huella hídrica para un cultivo de pitahaya amarilla (Selenicereus megalanthus). Publicaciones e Investigación, (9), 145-156. 
